Volume (4) No. (2) 2021

التعليم الإلكتروني كمتطلب لمهارات القرن الحادي والعشرين وتدريب معلمدي الرياضيات

أ.د. شيرين صلاح عبد الحكيم 
التعليم الإلكتروني كمتطلب لمهارات القرن الحادي والعشرين وتدريب معلمي الرياضيات

$$
\text { أ.د. شيرين صلاح عبد الحكيم }
$$

أستاذ المناهج وطرق تدريس الرياضيات، كلية البنات، جامعة عين شمس، مصر صلرين

shreen.salah@yahoo.com

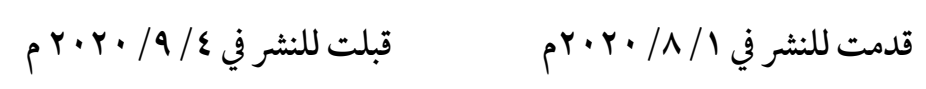

الملخص: هدف هذا المقال إلى التعرف علن تحديات القرن الحادي والعشرين ومهارات القرن الحادي والعشرين وأهم تصنيفاتها، وكيف يكسب الطالب المعلم مهار ات القرن الحلادي العشرين. وأحدها هو التعليم الإلكتروني و كذلك تقديم أهم الأدوار المتوقعة للمعلم في ضوء اكتساب مهارات القرن الحادي و العشرين. وما يترتب علن ذلك كله من ضرورة وأهمية تطوير الأداء التدريسي لمعلمي الرياضيات في

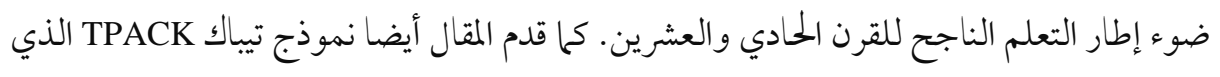
يؤكد علن التكامل بين المعرفة بالتكنولوجيا ومحتوى المادة الدراسية مع المعرفة بطرق التدريس

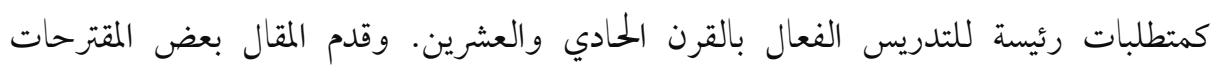

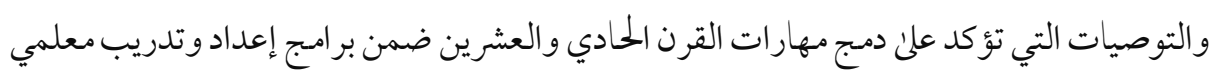
الرياضيات قبل وأثناء الخدمة.

الكلمات المفتاحية: التعليم الإلكتروني، مهارات القرن الحادي والعشرين، تدريب معلمي الرياضيات. 


\title{
E-Learning as a prerequisite of 21st century skills and training of mathematics teachers.
}

\author{
Prof. Dr. Shereen Salah Abd El-Hakeem
}

Professor of Mathematics Education, Women's college Ain shams university, Egypt

\section{shreen.salah@yahoo.com}

\section{Received in 1st August 2020}

Accepted in 4th September 2020

\begin{abstract}
The present article aims at identifying the challenges and the skills of $21 \mathrm{st}$ century; and how can the student teachers acquire these skills. What are the expectations of these students in the light of the 21 st century skills? So, educators should develop the teaching performance of math student teachers. The present article also presents TPACK model which assures the integration between technology and subject content as prerequisites for 21 st century effective teaching. Besides, the recommendations of embedding the 21 st century skills in pre and during preparing programs for math teachers.
\end{abstract}

Keywords: E-Learning, skills of the 21st century, mathematics teachers training, Professional Development. 
برزت في بداية القرن الحادي والعشرين تطورات سريعة ومتتابعة بسبب التغيرات المتعددة في قطاع الاتصالات والتكنولوجيا والتي أثرت في أغلب جوانب الحياة حتى أصبحت عصبها الرئيسي،

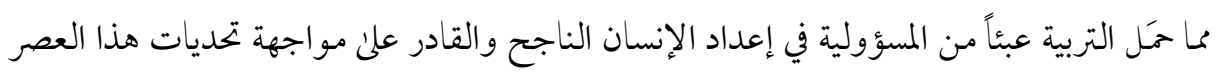
والتكيف مع تلك التطورات. ولقد نالت حركات تطوير التعليم اهتحام معظم دول العاله، حيث اعتبرته الحل الأساسي لِّي لأغلب المشكلات الناتجة عن التغيرات المحتملة لعولمة الحياة، ومن وسائل ذلك إعداد وتدريب وتأهيل معلم متمكن يستطيع مو اجهة تطورات هذا العصر المتجدد، وتوجيه قدرات وطاقات طلابه إلى تعرف المشكلات الحديثة والتعامل معها، فجودة المنظومة التعليمية مرهونة بالدرجة الأولن بجودة المعلم. وإذا كان المعلم الكفء يعتبر ضرورة لمواجهة تحديات المستقبل وإصلاح التعليم، فإنه يعتبر أمراً لازماً للرياضيات وتدريسها، حيث تعتبر الرياضيات أكثر العلوم مسايرة لتطورات العصر.

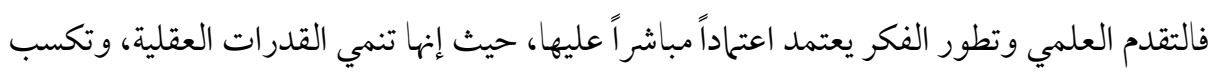
متعلمها المهارات الرياضية اللازمة للمقررات الأخرىن، كما أنها العصب الرئيسي لأغلب التقدمات النظرية والعملية، لأنها تؤثر في حل أغلب المشكلات العصرية. وعطفاً علن هذه التحديات ومـا تنتجه من آثار ومخاطر، يظل معلم الرياضيات أحد حلول تخفيفها، بحيث هو القادر على تمكين خخرجات التعليم من المهارات والخبرات اللازمة للنجاح في القرن الحادي والعشرين. لم تعد الطرق التقليدية في التدريس مجديةً، فعمليات التعلم والتعليم تجاوزت الكتاب المدرسي والوسائل التقليدية إلى وسائل عصر العولمة والسرعة والتكنولوجية، ومهارات معلم القرن الماضي لا تتناسب مع معطيات دور معلم القرن الحادي والعشرين الذي تجاوز دوره في نقل المعلومات و المعرفة إلى إعداد متعلم قادر علئ النجاح في القرن الحلاي.

ما تحديات القرن إب؟ ولماذا؟ 


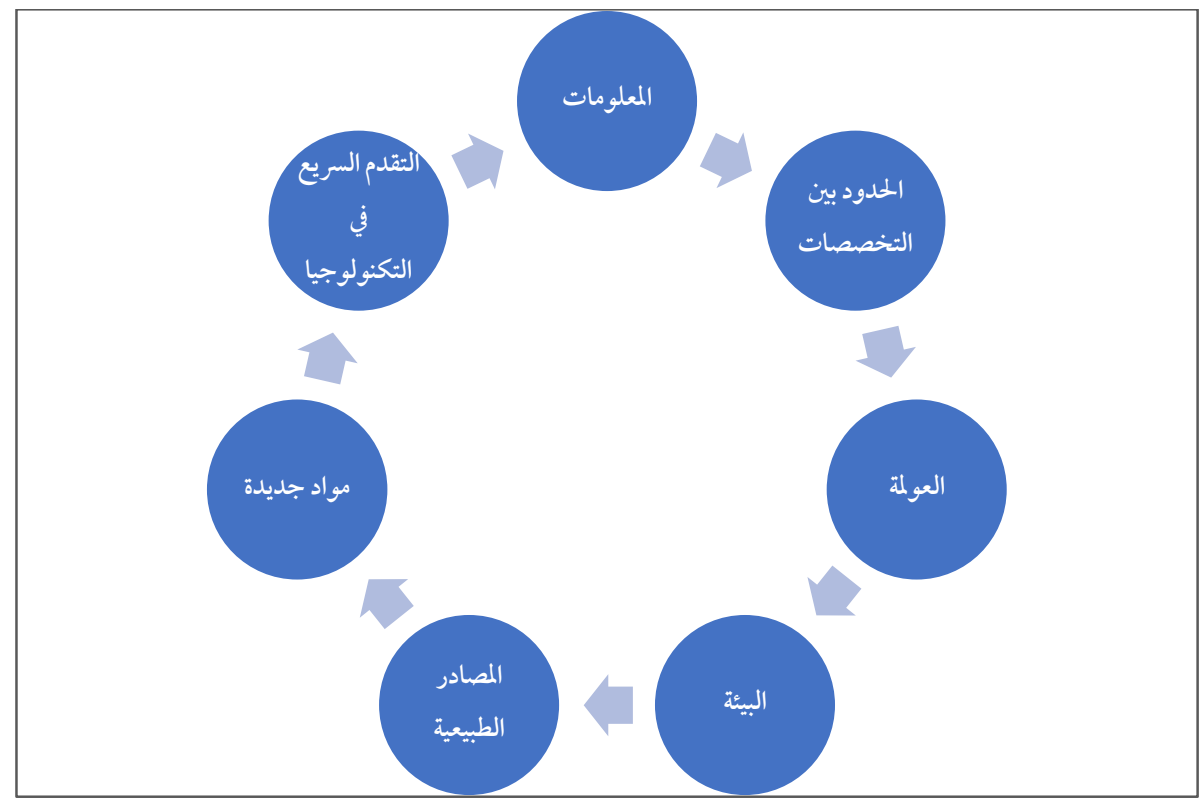

$$
\begin{aligned}
& \text { شكل (1) سمات القرن الحادي و العشرين }
\end{aligned}
$$

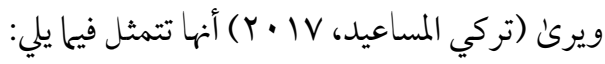

$$
\begin{aligned}
& \text { • تحديات العلوم والتكنولوجيا. } \\
& \text { • تحديات التنمية الاقتصادية والاجتماعية. }
\end{aligned}
$$

• تحديات تطوير التعليم. فلم يعد التعليم يقتصر علن التدريب علن المهارات الأساسية (اللغة الأم والرياضيات والعلوم)، بل أصبحت النظم والسياسات التربوية تسعي إلى تمكين الطلبة من كفاءات رئيسية تؤهلهم للتعلم و العمل في المستقبل وذلك من خلال:

$$
\text { م التو اصل بل اللغة الأم. }
$$

م الكفاءات الأساسية في الرياضيات والعلوم والتكنولوجيا.

$$
\text { م الكفاءة الرقمية. }
$$$$
\text { م روح المبادرة وريادة الأعمال. }
$$$$
\text { م الوعي الثقافي والقدرة على التعبير. }
$$

\section{http://dx.doi.org/10.29009/ijres.4.2.3}


م الكفاءة الاجتماعية والمدنية وتعلم كيفية التعلم.

o التفكير الناقد والإبداع وحل المشكلات واتخاذ القرارات وإدارة المشاعر وتقييم المخاطر.

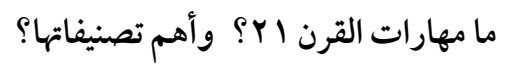

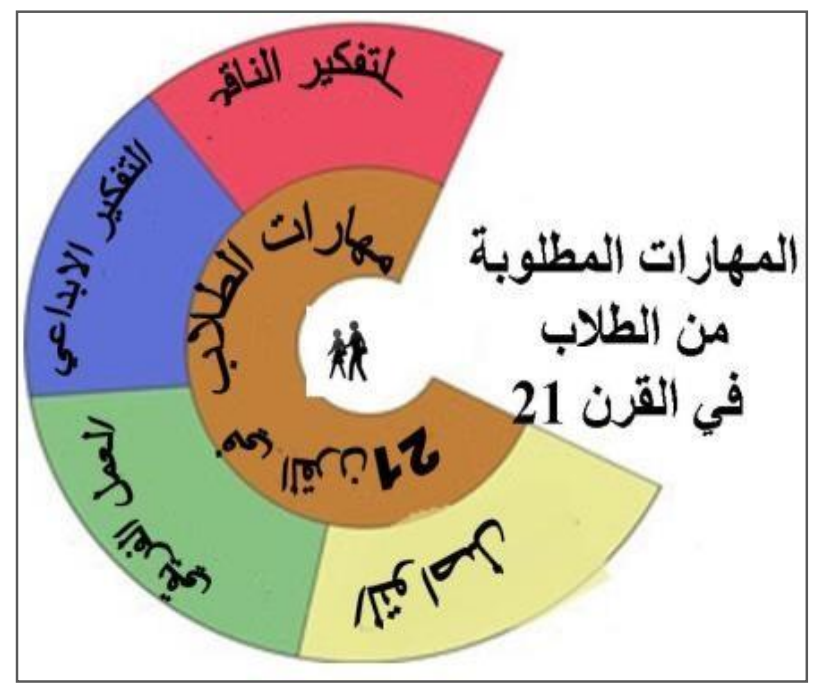

$$
\text { شكل (r) مهارت القرن الحادي والعشرين }
$$

يذكر (صفوت خليل، 10 • ب) أن مهارات القرن الحلادي والعشرين هي المعنية" بالإنتاج المباشر للشراكة بين التربويين وقطاع الاقتصاد وأصحاب القرار السياسي من أجل بناء إطار فكري للتعليم القومي بهدف تطوير وبناء نموذج لنظم التعليم من الروضة وحتى الجحامعة، وقد اتبع هذا النظام في العديد من الدول مثل الولايات المتحدة الأمريكية وكندا وإنجلترا.

\section{وتصنفها (نوال شلبي، ع ( • ) كما يلي:}

• مهار ات العصر الرقمي Digital Age Literacy، وهي مهارات ضرورية للحياة والعمل في بجتمع المعرفة وتتمثل في القدرة علن استخدام التكنولوجيا الرقمية وأدوات الاتصال والشبكات وصو لا إلى المعلومات وإدارتها وتقويمها وإنتاجها. وتشمل: الثقافة الأساسية - 
الثقافة العلمية - الثقافة الاقتصادية - التكنولوجية البصرية والمعلوماتية - فهم الثقافات

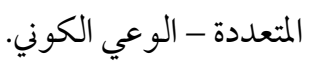

مهارات التفكير الإبداعي Inventive Thinking وتشمل القدرة علن التكيف و إدارة التعقيد - التوجيه الذاتي - حب الاستطلاع - الإبداع -تحمل المخاطر - مهارات التفكير العليا والتفكير السليم.

مهارات الاتصال الفعال Effective Communication وتشمل مهار ات العمل في فريق المهارات الشخصية - المسؤولية الشخصية والاجتماعية والمدنية - الاتصال التفاعلي. مهارات الإنتاجية العالية High Productivity وتشمل مهار ات تحديد الأولويات - مهيدي التخطيط والإدارة وصولا إلى تحقيق النتائج - الاستخدام الفعال للأدوات التكنولوجية في

$$
\text { العاله الو اقعي للتو اصل والتعاون وحل المشكلات وإنجاز المهام. }
$$

مما سبق يتضح أهمية التعليم الإلكتروني كأحد المتطلبات اللازمة لمهارات القرن الحادي والعشرين، وفيلا يلي مزيد من التوضيح لمذا المتطلب الهام المعاصر:

$$
\text { تعريف التعليم الإلكتروني: (زيتون، ه • ( ) }
$$

التعليم الإلكتروني هو نظام تفاعلي للتعليم يقدم للمتعلم باستخدام تكنولوجيات الاتصال

والمعلومات، ويعتمد علن بيئة إلكترونية رقمية متكاملة تعرض المقررات الدراسية عبر الشبكات

الإلكترونية، وتوفر سبل الإرشاد والتوجيه وتنظيم الاختبارات وكذلك إدارة المصادر و العمليات

وتقويمها.

تكمن أهمية التعليم الإلكتروني في حل مشكلة الانفجار المعرفي والإقبال المتزايد علن التعليم

وتوسيع فرص القبول في التعليم، إضافة إلى التمكين من تدريب وتعليم العاملين دون ترك أعمالهم

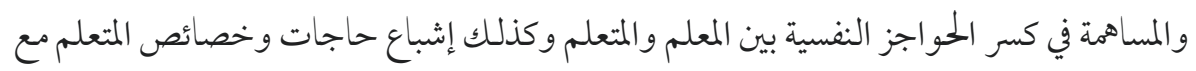

رفع العائد من الاستثمار بتقليل تكلفة التعليم 


$$
\text { أهداف التعليم الإلكتروني: (بو الفلفل، با • Y ) }
$$

تو فير مصادر متعددة ومتباينة للمعلومات تتيح فرص المقارنة والمناقشة والتحليل والتقييم.

إعادة هندسة العملية التعليمية بتحديد دور المُعلم والمُعجلم و المؤسسة التعليمية.

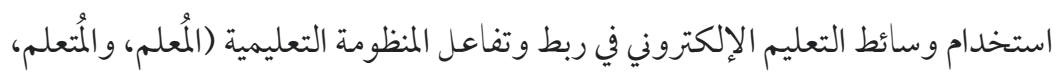

$$
\text { والمؤسسة التعليمية، والبيت، و المجتمع، والبيئة). }
$$

نمذجة معيارية التعليم. - مان.

تبادل الخبرات التربوية من خلال وسائط التعليم الإلكتروني.

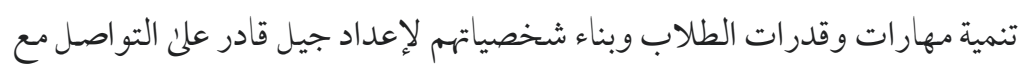

الآخرين وعلنم التفاعل مع متغيرات العصر من خلال الوسائل التكنولوجية الحميثة.

نشر الثقافة التكنولو جية بـا يساعد في خلق مجتمع إلكتروني قادر على مو اكبة مستجدات

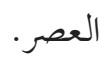

ويشار إلى أن تحقيق ذلك يتطلب التهيئة لذلك من خلال ما يلي علن سبيل المثال:

توفير البنئ التحتية اللازمة، المتمثلة في الشبكات والأجهزة والبريجيات.

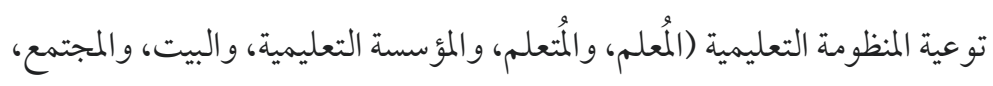

و البيئة)، بأهمية وكيفية وفعالية التعليم الإلكتروني، لخلق التفاعل بين هذه المنظومة.

تدريب (المُعلم، المتُعلم) بما يمكن تسهيل استخدام هذه التكنولوجية.

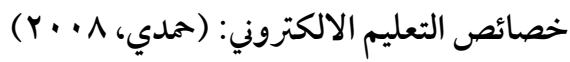

توفير جميع وسائل التفاعل الحي بين الطالب والمدرس وإمكانية تفاعل الطلبة والمدرس على المدي

$$
\text { السبورة الالكترونية. }
$$

تفاعل الطالب مع المدرس بالنقاش حيث يمكن للطالب التحدث من خلال الميكروفون

$$
\text { المتصل بالحاسب الشخصي الذي يستخدمه. }
$$


تمكين المدرس من عمل استطلاع سريع لمدا تجاوب وتفاعل الطالب مع نقاط الدرس المختلفة والتي تعرض على الهواء.

تمكين المدرس و الطالب من عمل تقييم فوري لمدا تجاوب الطلبة من خلال عمل استبيان سريع وفوري يستطلع من خلاله المدرس مدا تفاعل الطلبة معه ومع محتوى المادة التعليمية و التربوية.

يمكن للمدرس عمل جولة للطلبة لأحد المو اقع التعليمية المتاحة على الانترنت تمكين المدرس من استخدام العديد من وسائل التعليمية التفاعلية المختلفة مثل مشاركة التطبيقات

مساعدة المدرس على تقسيم الطلبة إلى بجموعات عمل صغيرة في غرف تفاعلية بالصوت

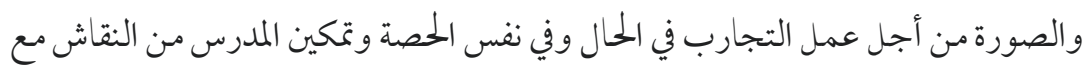
أي من مجموعات العمل ومشاركة جميع الطلبة في تحليل نتائج أحد بجموعات العمل. تمكين المدرس و الطالب من عمل تقييم فوري لمدا تجاوب الطالب من خلال اختبار سريع يتم تقييم ومناقشة تفاعل الطالب معه في الحال وفن وجود المدرس.

$$
\text { أنواع التعليم الإلكتروني }
$$

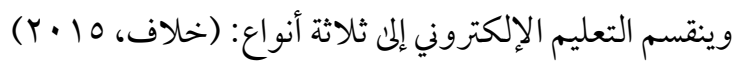

1- التعلم الإلكتروني المتزامن (Synchronous E-learning)

ويتم online وبطريقة مباشرة وبث حي مع المتعلمين يتزامن فيه وقت إلقاء المحاضرات مع وجود المعلم والطالب أمام شاشات الحاسوب، ويتمكن الطرفان من المناقشة والحوار وطرح الأسئلة وتلقي التغذية الراجعة feedback، ويكون ذلك عبر غرف محادثة أو من خلال تلقي الدروس عبر ما يعرف بالفصول الافتراضية (virtual classroom)، ويحتاج هذا النوع إلى سرعة عالية لإِنترنت. 
r- التعلم الإلكتروني غير المتزامن (Asynchronous E-learning) ويتم التفاعل فيه بشكل غير مباشر ويستخدم عندما يكون النت بطيئا أو متقطعا مثل المتتديات التعليمية.

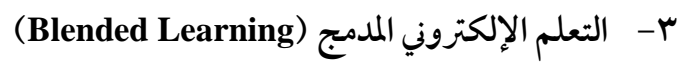
يندمج فيها التعليم الإلكتروني مع التعليم الصفي (التقليدي) في إطار واحد.

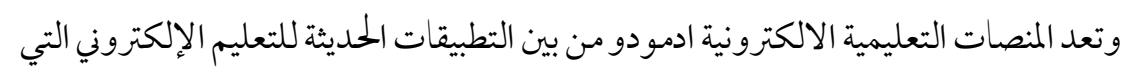
تساعد علن التعليم والتعلم الجيد، من خلال إيجاد بيئة تعلمية تتفق ومتطلبات العصر الرقمي، فهي إحدى تطبيقات ويب • , r، وتتميز ادمودو Edmodo بسهولة الاستخدام، وتعزز التفاعل بين المعلم والمتعلمين، من خلال توفير بيئة تربوية آمنة ومناسبة لتنمية التفكير والإبداع، وتحقيق نواتج التعلم

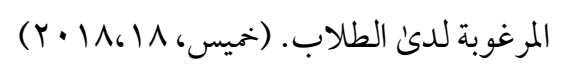
توظيف التعليم الإلكتروني في التدريس تتم الاستفادة من التعليم الإلكتروني في التدريس بتوظيفه بعدة طرق لوقي 1- النموذج المساعد (المكمل)يستخدم بعض تقنيات التعليم الإلكتروني كتدعيم للتعليم

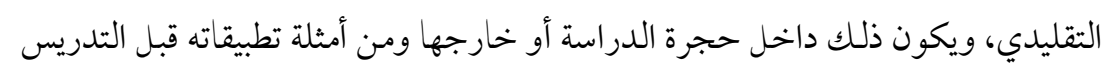
يوجه المعلم الطالب للاطلاع علن درس معين علن شبكة الانترنت أو على قرص مدمج، قيام المعلم بتكليف الطلاب بالبحث عن معلومات معينة في شبكة الانترنت.

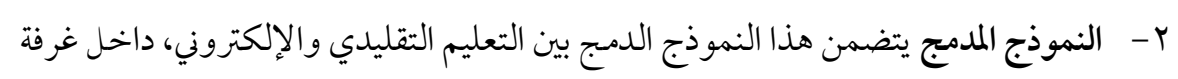
الدراسة أو الأماكن المجهزة بتقنيات التعليم الإلكتروني، ويمتاز بالجمع بين مزايا التعليم

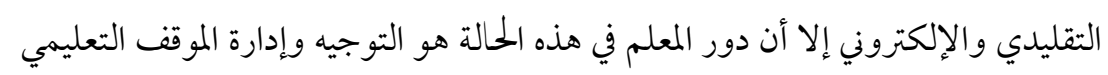

$$
\text { والمتعلم يكون دوره ايجابي. }
$$

r- النموذج الخالص يستخدم التعليم الإلكتروني بديلا للتعليم التقليدي بحيث يتم التعلم من أي مكان وفي أي وقت من قبل المتعلم، تعمل الشبكة كوسيط أساسي لتقديم كامل عملية 
التعليم.، ومن أمثلة تطبيقاته الدراسة الذاتية المستقلة (يدرس الطالب المقرر الإلكتروني انفر اديا). أن يتعلم الطالب مع مجموعة زملاعه، من خلال درس أو انجاز مشروع بالاستعانة بأدوات التعليم الإلكتروني التشاركية مثل غرف المحادثة والمنتديات.

$$
\text { كيف نكسب الطالب المعلم مهارات القرن آج؟ }
$$

يؤكد (ساما خميس، 1 ( • ( ) علن أنه يمكن إكساب الطالب المعلم هذه المهارات من خلال

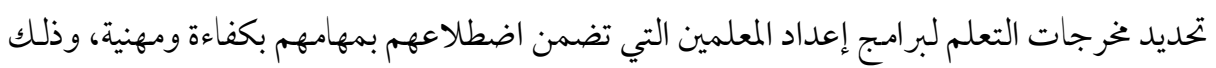
بامتلاك الخريج القدرة على أن:

ا. يقدم نموذجا تعليميا لجميع مواد المحتوى الأكاديمي المعرفي، ويظهر فها للموضوعات

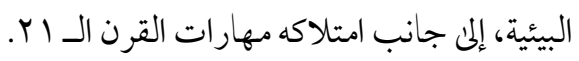
r. يستخدم مدنى واسعا من استراتيجيات التقييم لتقويم معرفة ومهارات القرن الـ ال ب لدني المتعلمين.

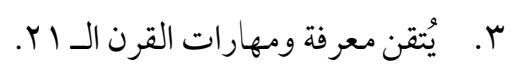

ع. يعمل ميسرا للتعلم بوضع المتعلمين في بؤرة الاهتمام، باستخدام استراتيجيات التدريس المختلفة (بها فيها الاستخدام الملائم للتكنولوجية)، والتي تمكن المتعلمين من العمل في بجموعات، واتخاذ القرارات الجماعية، والوصول إلى اتفاق جماعي في الرأي والعمل معا لـا

$$
\text { لتحقيق الأهداف التي تم الاتفاق عليها. }
$$

ه. يُخطط ويقدم الدروس والوحدات التي تربط المفاهيم والمهارات الأكثر أهمية والتي يجتاج المتعلمون لمعرفتها والعمل بها، وذلك يعني تجنب التعليم غير المنظم، والحقائق المنفصلة غير تهري

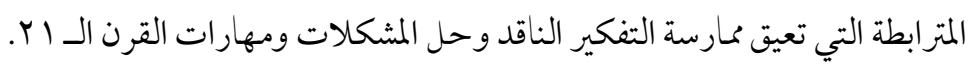
T. يتابع فرص التطوير المهني لمهارات القرن الـ اب في أثناء العمل، ويعمل زميلا مدربا وموجها للمعلمين المبتدئين. 
V. يتكيف بمرونة ومسؤولية وكفاءة؛ بناء علن معرفته بالعوامل المؤثرة علن البيئة والصحة

$$
\text { و الاقتصاد، و اندماجه مع متطلبات المجتمع المدني والعامر. }
$$

^. يستخدم التكنولوجية بمهارة وسلاسة وبطرق ملائمة للتدريس في جميع أنواع المحتوىن

$$
\text { المعرفي، في الممارسات اليومية. }
$$

9. يتفهم ويعمل محاميا ومؤيدا ومعززا للاحتياجات الانفعالية والجسمية والتعليمية لجميع المتعلمين.

$$
\text { ما الأدوار المتوقعة للمعلم في ضوء اكتساب مهارات القرن ال }
$$

وحول تلك الأدوار المتوقعة للمعلم الواعي مستقبليا يذكر (صفوت خليل، 10 • ب) ضمن

$$
\text { مهارات القرن الحادي و العشرين محددا إياها فيا يلي: }
$$

أ- أد أدوار مجتمعية (Societal Roles)

• مو اصلة الإسهام في نقل ثقافة المجتمع إلى الأجيال الجلديدة (المتعلمين) .

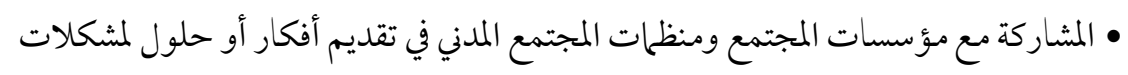

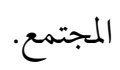

• تبني موقف سياسي مرتكز علن رؤية واضحة للقضايا السياسية الداخلية والخارجية

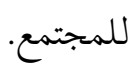

$$
\text { • • تبني توجه ثقافي قائم علن الوعي بقضايا العالم. }
$$

• المشاركة في مجمل الجهود المبذولة في مؤسسات المجتمع في التنمية البشرية.

ب- ب أدوار مهنية (Professional Roles)

• الانتحاء إلى مهنة التعليم من خلال العضوية العاملة في المنظمات المهنية التعليمية.

$$
\text { • • •حمل المسؤولية الشخصية عن نموه المهني المستمر. }
$$




$$
\text { • تبني موقف أو توجه واضح من المشكلات التعليمية في المجتمع. }
$$

ج- أدوار أكاديمية (Academic Roles) • بناء قاعدة معلومات تتسم بالعمق والشمول والحداثة في بجال تخصصه العلمي.

$$
\text { • توظيف محتوئ التخصص في حل المشكلات الاجتماعية. }
$$

• تحمل مسؤولية ذاتية عن متابعة التطور المستحدث في محتوئ مادة تخصصه.

$$
\text { • الإسهام في إنتاج المعرفة في بجال تخصصه. }
$$

\section{د- الأدوار التعليمية (Instructional Roles)}

• المشاركة في الخطط التعليمية.

$$
\text { • تيسير (تسهيل) تعلم الطلبة بطريقة مرنة وإبداعية. }
$$

• • تبني توجه يقوم علن قناعة بقدرة المتعلم علنى التنظيم الذاتي لتعلمه.

$$
\text { • دمج تكنولوجية المعلومات والاتصال في التعليم. }
$$

• تحمل المسؤولية الذاتية في الدعم المستمر لمهاراته في تطبيق أدوات تكنولوجية المعلومات

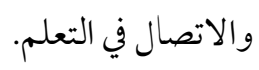

وبهذا تتضح أهمية الأداء التدريسي لمعلمي الرياضيات، وتطويره في ضوء إطار التعلم الناجح

للقرن الحادي والعشرين، بطرح مداخل تدريسية حديثة تشكل المنطلقات والمسلمات التي تحقق هذا التعلم الناجح، بحيث يشمل هذا التطوير التفاعل بين مكونات تدريس الرياضيات وفق ثلاثة محاور كالتالي:

تخطيط التدريس: ويشمل عدة إجراءات من أهمها: تحديد نواتج التعلم المتوقعة، واختيار طرق تدريسية وتقنيات تعليمية وأساليب تقويمية فعالة، بها يتناسب وتحقيق أهداف التعلم الناجح في إطار التعلم الناجح للقرن الحادي و العشرين. 
تنفيذ التدريس: ويشمل عدة إجراءات من أهمها: تطبيق طرق تدريسية محقة للتعلم الناجح في إطار التعلم الناجح للقرن الحادي والعشرين، مع التدرج في التدريس بها يلائم مراحل التدريس البنائي.

تقويم التدريس: ويشمل عدة إجراءات من أهمها: استخدام أساليب تقويمية متنوعة لقياس وتقويم مهارات القرن الحادي والعشرين، كملفات الإنجاز (Portfolio) و الملاحظة وتقويم الأقران والتقويم الذاتي، مع التأكيد علن مشاركة الطلاب في عملية التقويم وإجراءاتها، و تقديم التغذية الراجعة المستمرة لكافة أعمال ومشار كات الطلاب.

ولتحقيق ذلك ينبغي التركيز علن تطوير طرق تدريسية تعين معلمي الرياضيات على تحقيق

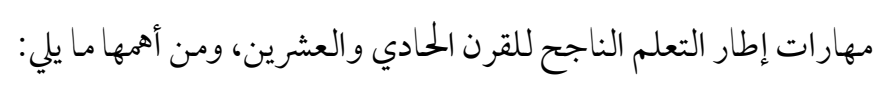

استراتيجيات تعليم وتعلم مهارات التعلم والابتكار ومنها: حل المشكلات، العصف الذهني، خر ائط التفكير، القبعات الست، الخرائط الذهنية، برنامج الكورت لتعليم التفكير،

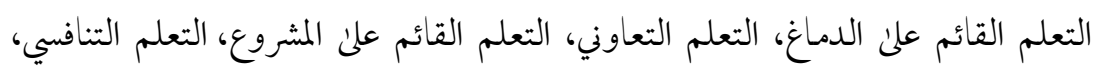
المناقشة. استراتيجيات تعليم وتعلم مهارات الحياة والتكيف ومنها: التعلم القائم علئ المشروع، التعلم التعاوني، التعلم التشاركي، الخرائط المعرفية، الاستقصاء، الاستكشاف، لعب الأدوار. استراتيجيات تعليم وتعلم مهارات المعلومات والإعلام والتكنولوجيا ومنها: الرحلات المعرفية عبر الويب، المحاكاة، الاستقصاء، الاستكشاف، الخرائط الإلكترونية، التعلم التعاوني الإلكتروني، المناقشة الإلكترونية، العصف الذهني الإلكتروني. كما ينبغي تنظيم برامج تدريبية متطورة لتدريب معلمي الرياضيات علن مهارات التدريس في إطار التعلم الناجح للقرن الحادي والعشرين، وعقد ورش تعليمية، ولقاءات وندوات علمية لتوعية معلمي الرياضيات بأهمية التدريس في ضوء مهارات إطار التعلم الناجح للقرن الحادي و العشرين، علن 
أن يتضمن ذلك تطبيقات تدريسية لمهارات التعلم والابتكار، والحياة والتكيف، والمهارات الإعلامية، واستخدام التقنيات بفاعلية في التدريس، مع التأكيد على تطوير البيئة المدرسية. إن متطلبات القرن الحادي والعشرين تفرض علن النظم التعليمية المختلفة تزويد الطلاب بمجموعة من المهارات المختلفة اختلافاً جذرياً عن تلك التي كانت سائدة في العقود السابقة. وبشكل أكثر تحديداً فإن هذه المتطلبات تفرض علن المدارس أن تركز علن ما يعرف بمهارات القرن الحادي و العشرين. وفي هذا الصدد، حددت منظمة الشر اكة من أجل مهارات القرن الحادي والعشرين -وهي منظمة مؤلفة من شركاء تجاريين وصناع سياسة ومعلمين- بعض الإرشادات الخاصة بالمهارات الضرورية للمتعلمين المستقبلين. وقد تضمنت هذه الإرشادات بجموعة من المهارات والتي شملت التفكير الناقد، والتعاون، وإصدار الأحكام، والتنور المعلوماتي، والتنور الوسائطي، والتعامل مع التطبيقات التكنولوجية، والمهارات الاجتماعية والعبر ثقافية، والقيادة والمسؤولية، والتنور الاقتصادي والتجاري، والكفايات الرقمية، والمرونة والقابلية للتكيف، والمبادأة والتوجه الذاتي، وفهم القضايا الكونية. ولكي نتمكن من تنمية هذه المهارات بفاعلية لدئ الطلاب، فإنه يتعين أن يتو افر لدينا معلمون يتسمون بخصائص وسمات المعلم الفعال في القرن الحادي والعشرين حتى تكون هناك مخرجات تعليمية ذات جودة عالية بالنسبة للمعلم وللمتعلم علن حد سواء. ومن بين أكبر خصائص وسمات المعلم الفعال تلك القدرة على توظيف التكنولوجية بفاعلية. ونظراً للدور الحيوي الذي تلعبه التكنولوجية في عصرنا الحلاي في إطار مهارات القرن الحادي و العشرين، فإنه من الأهمية بمكان أن يكون هناك إطار عمل جديد يساعد علن فهم وتقويم المعارف و المهارات التي يحتاجها المعلمون لتوظيف التكنولوجية بفاعلية في المحتون الدراسي، حيث إن امتلاك المعلم لبعض المهارات التكنولوجية لا يضمن الاستخدام الأمثل لها في العملية التعليمية والتعلمية. 
وبالرغم من إدراك الأهمية البالغة لاستخدام تكنولوجيا التعليم، وإعداد المعلمين لاستخدام التكنولوجية في حجرات الدراسة، إلا أن بعض الدراسات أظهرت فشل التقنيات التعليمية بأقصن إمكانياتها في إحداث تحو لات جذرية في العملية التعليمية من خلال بعض الحالات. كما أن التدريب علن استخدام التكنولوجية قد أصبح بمثابة مكون هام في كل من برامج تدريب المعلمين سواءً قبل أو أثناء الحدمة. ومع ذلك فإن في أغلب الأحيان، يركز التدريب على التكنولوجية في حد ذاتها بدلاً من التركيز علن توظيف التكنولو جية في مادة التخصص التي يقوم المعلمون بتدريسها. ولكي يتم استخدام التكنولوجية علن نحو ناجح في العملية التعليمية، لابد للمعلمين أن يمتلكوا المعرفة التكنولوجية، بالإضافة إلى المعرفة بمحتوى التخصص، والمعرفة بطرق التدريس والربط بين هذه المعارف عند التخطيط للتدريس. من الناحية التاريخية، ركز إعداد المعلم علن تنمية المعرفة بمحتوى مـادة التخصص لدىن المعلمين، جنباً إلى جنب مع المعرفة بطرق تدريس هذا المحتوى وهو ما استند بالأساس إلى نموذج "شو لمان". وقد اقترح "شولمان" تصنيف المعرفة اللازمة للمعلمين إلى بجالين متمايزين و هما المعرفة بمحتوىن المادة الدراسية subject matter content knowledge والمعرفة بطرق تدريس هذا pedagogical content knowledge. المحتوئ واستناداً إلى هذا النموذج التاريخي الر ائد والذي وجه عمليات إعداد المعلمين بكليات التربية لفترة طويلة، قام “ميشرا وكوهلر بإضافة ضلع ثالث لثنائية المعرفة بمحتوى مادة التخصص، و المعرفة بطرق التدريس، وهي المعرفة باستخدام تقنيات التعليم الأمر الذي أثمر عن نموذج أكثر شمولاً لتحديد خصائص ومهارات ومعارف المعلم الفعال بـا يتلاءم أكثر مع متطلبات القرن الحادي والعشرين، وقد أطلق علئ هذا النموذج المعدل مسمى نموذج تيباك. 
Prof. Dr. Shereen Salah Abd El-Hakeem

Volume (4) No. (2) 2021

برز نموذج تيباك TPACK وهو أحد النماذج المعاصرة والذي يؤكد علن التكامل ما بين المعرفة بالتكنولوجية والمعرفة بمحتوئ المادة الدراسية جنباً إلى جنب مع المعرفة بطرق التدريس كمتطلبات رئيسة للتدريس الفعال باستخدام التقنيات التعليمية.

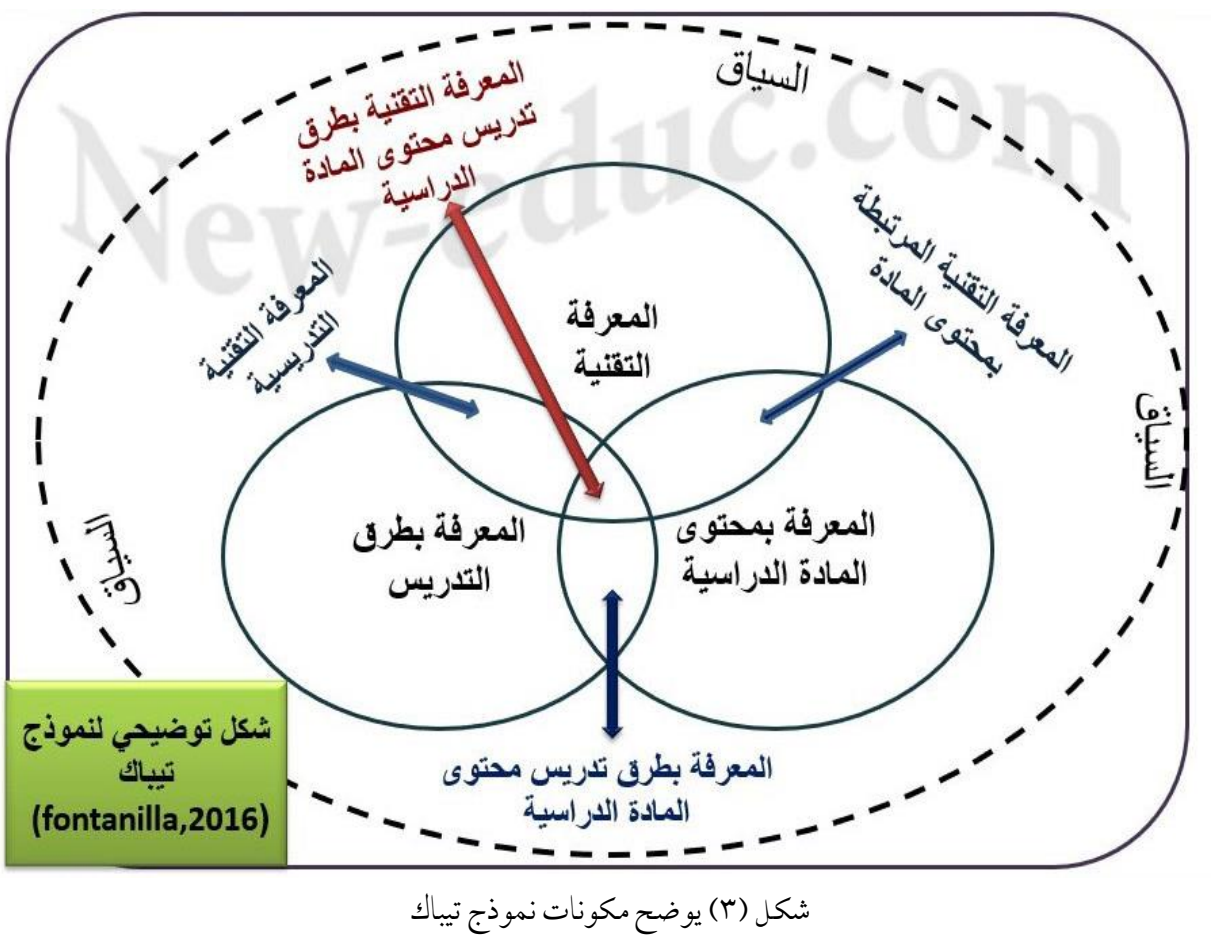

إن المعرفة التكنولو جية المتعلقة بطرق تدريس محتوئ مادة التخصص TPACK تتألف من سبعة بجالات رئيسة ونذكرها بشكل موجز فيها يلي: ا - المعرفة التكنولوجية:TK Technology Knowledge إذ يتعين علن المعلمين فهم تقنيات المعلومات والاتصالات بشكل كافِ لكي يمكنهم تطبيقها بفعالية في حجرات الدراسة وأن تكون لديهم أيضاً المعرفة بكيفية تعديل الغرض من التقنيات بحيث يمكن استخدامها علن نحو أفضل. وتنطوي هذه المعرفة علن الإلمام بمختلف التقنيات 
المتنوعة التي قد يستخدمها المعلم في حجرة الدراسة سواء التقنيات الرقمية الحديثة مثل الهو اتف النقالة والوسائط المتعددة أو التقنيات التقليدية.

PK Pedagogical Knowledge ب المعرفة التربوية

والتي تتضمن المعرفة بطبيعة المتعلمين، استراتيجيات تقويم الطلاب، مراعاة الفروق الفردية بين المتعلمين أثناء التدريس، إدارة الصف، تقديم التغذية الراجعة وتقييم فهم الطلاب لما يتم تعليمه

بشكل مستمر.

ب- م- المعرفة بمحتوى مادة التخصص CK Content Knowledge وتتضمن المعرفة بالمفاهيم، النظريات، النهاذج وأطر العمل المفاهيمية للتخصص. مثل توظيف المعلم لطرق التفكير الأساسية في مادة التخصص وإثرائه للمحتوى بمواد علمية إضافية. ع - المعرفة التكنولوجية المتعلقة بمحتوى مادة التخصص TCK Technological Content Knowledge وتتضمن معرفة المعلم بالتقنيات المعينة والملائمة لتعلم المادة الدراسية في تحصصه، عرض محتون المادة الدراسية باستخدام إمكانيات الوسائط المتعددة ومساعدة الطلاب علئ استخدام التكنولوجية للبحث عن مصادر معلومات مرتبطة بالمحتوىن.

هـ المعرفة التكنولوجية التربوية TPK Technological pedagogical Knowledge من خلال توظيف المعلم لأدوات التكنولوجية بطرق واستراتيجيات التدريس المناسبة للتخصص الذي يقوم بالتدريس فيه، وأيضا توظيف التكنولوجية بشكل مناسب في تقييم أداء الطلاب ونو اتجهم التعليمية.

جCK Pedagogical content Knowledge المعرفة بطرق تدريس محتوى مادة التخصص وتتضمن هذه المعرفة، التدريس، التعلم، المنهج والتقويم ومن الأمثلة عليها المعرفة بأساليب الدمج و التكامل بين المحتوكأ وطرق التدريس لتحقيق ممارسات أفضل للعملية التعليمية. 
TPCK Technological المعرفة التكنولوجية المتعلقة بطرق تدريس محتوى مادة التخصص - V

\section{Pedagogical Content Knowledge}

وتتجسد هذه المعرفة من خلال التكامل بفاعلية أثناء تنفيذ التدريس بين المعرفة التكنولوجية، المعرفة بمحتوى مـادة التخصص و المعرفة بالأساليب التدريسية.

نؤكد علن ضرورة أن يكون هناك تغيير جذري في نظم إعداد وتأهيل معلمي الرياضيات بالجامعات بحيث تتضمن إضافة التكنولو جية باعتبارها بعداً ثالثاً لا يتجزأ من أبعاد إعدادهم وتنمية مهار اتهم واتجاهاتهم المهنية والاستفادة من النماذج المعاصرة في ذلك.

$$
\text { المقترحات والتوصيات }
$$

دم-ج مهارات القرن الواحد والعشرين ضمن مقررات لوائح إعداد معلم الرياضيات قبل

$$
\text { وأثناء الخدمة. }
$$

تطوير برامج التنمية المهنية لمعلم الرياضيات بما يتوافق مع متطلبات ومستجدات العصر الحالي.

الاستفادة من نتائج التجارب العالمية الرائدة في تطبيق تعلم معلم الرياضيات مدى الحياة

$$
\text { وتطوير أدائه المهني. }
$$

إعداد برامج تدريبية للطالب المعلم (تحصص الرياضيات) من أجل إكسابه هذه المهارات

$$
\text { والإبداع فيها. }
$$

تكليف الطالب بواجبات وأعحال منزلية تنمي مهارات القرن الواحد والعشرين لديه. وضع خطة تقويمية لأداء معلم الرياضيات قبل وأثناء الخدمة. 
آل كباس، عزة. V • بام. ورقة علمية بعنوان نموذج تيباك TPACK كأحد النماذج المعاصرة لتحديد

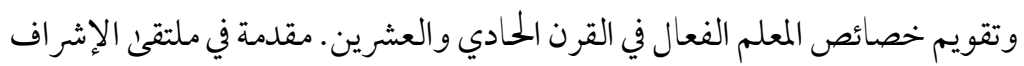

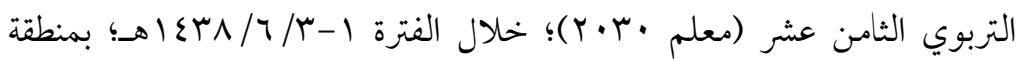

$$
\text { الحدود الشمالية. }
$$

بو الفلفل، ابراهيم وشهيب، عادل. (r| •Y). "واقع التعليم الالكتروني في الجامعة الجزائرية دراسة

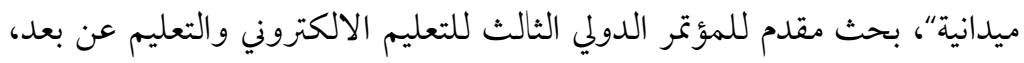

$$
\text { الرياض. }
$$

بيرز، سيو (عا +rم). تدريس مهارات القرن الحادي والعشرين أدوات عمل، (ترجمة محمد بلال

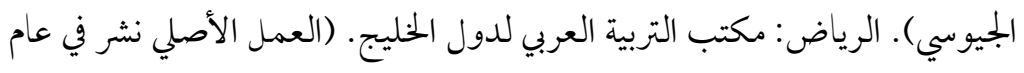

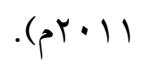

تريلنج، بيرني، وفادل، تشارلز (rا • بم) .مهارات القرن الحادي و العشرين: التطلم في زمنتا، (ترجمة بدر عبدالله الصالح). الرياض: جامعة الملك سعود، النشر العلمي والمطابع. (العمل

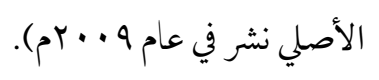

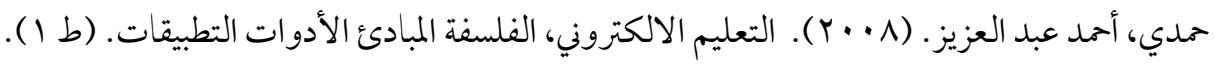
ع عان: دار الفكر.

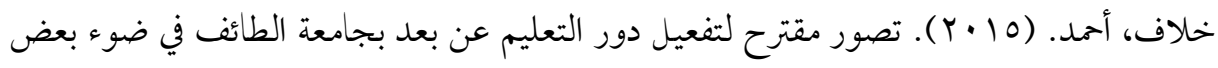

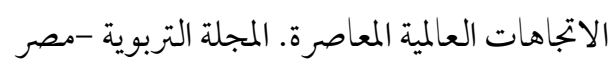

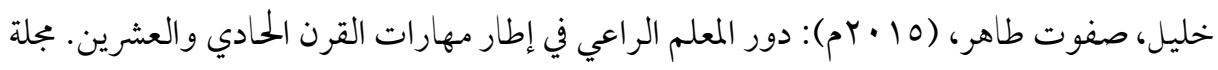

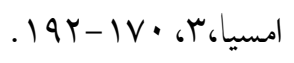

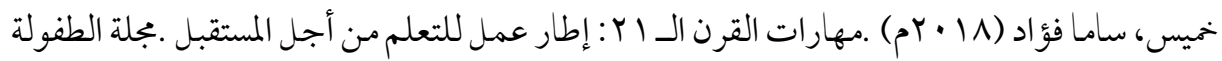

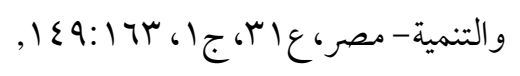


زيتون، حسن حسين. (0 · · ). رؤية جديدة في التعلم الإلكتروني: المفهوم، القضايا، التطبيق، التقييم.

$$
\text { (ط (1) ). الرياض: الدار الصوتية للتربية. }
$$

شلبي، نوال محمد (ع ( • Y): إطار مقترح لدمجج مهارات القرن الحلادي والعشرين في مناهج العلوم بالتعليم الأساسي في مصر. المجلة التربوية الدولية المتخصصة، س(• (1)، ا-بr. القضاة، خالد يوسف ومقابلة، بسام. (r · ب). تحديات التعلّم الإلكتروني التي تواجه أعضاء الهيئة التدريسية في الجامعات الأردنية الخاصة، بجلة المنارة، المجلد التاسع، العدد بّ.

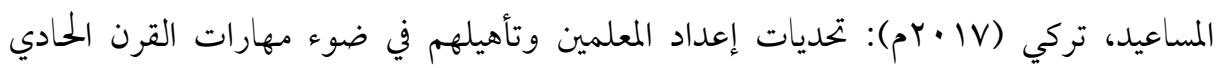
والعشرين. المؤتمر الرابع كلية التربية والعلوم الأساسية، عجمان - الإمارات العربية 


\section{References:}

Al Kabbas, Azza. 2017. A scientific paper entitled TPACK model as one of the contemporary models for identifying and evaluating the characteristics of effective teacher in the twenty-first century. Introduction to the 18th Educational Supervision Forum (Teacher 2030); During the period 1-3 / $6 / 1438 \mathrm{H}$; In the northern border region. (In Arabic).

Alkodah,, Khaled Youssef and an interviewer, Bassam. (2013). E-learning challenges facing faculty members in private Jordanian universities, Al-Manara Magazine, Volume Nine, Issue 3. (In Arabic).

Beers, Seo (2014 AD). Teaching the skills of the twenty-first century as work tools, (translated by Muhammad Bilal Al-Jayyousi). Riyadh: Arab Bureau of Education for the Gulf States. (The original work was published in 2011 AD). (In Arabic).

Bo Al-Felif, Ibrahim and Shihaib, Adel. (2013). "The reality of e-learning in the Algerian university is a field study", a paper presented to the Third International Conference on E-learning and Distance Education, Riyadh. (In Arabic).

Hamdi, Ahmed Abdel Aziz. (2008). E-learning, philosophy, principles, tools, applications. (I 1). Amman: House of Thought. (In Arabic).

Khalaf. Ahmed. (2015). A proposed conception to activate the role of distance education at Taif University in light of some contemporary global trends. The Educational Journal - Egypt. (In Arabic).

Khalil, Safwat Taher, (2015): The role of the pastor teacher in the framework of twentyfirst century skills. Emasia Journal, 3, 170-192. (In Arabic).

Khamis, Sama Fouad (2018). 21st Century Skills: A Framework for Learning for the Future. Childhood and Development Journal - Egypt, Vol. 31, Part 1, 149: 163. (In Arabic).

Shalaby, Nawal Mohamed (2014): A proposed framework for integrating twenty-first century skills into science curricula in basic education in Egypt. Specialized Educational International Journal, 3 (10), 1-33. (In Arabic). 
Trailing, Bernie, and Fadel, Charles (2013). 21st Century Skills: Learning in Our Time, (translated by Badr Abdullah Al-Saleh). Riyadh: King Saud University, Scientific Publishing and Press. (The original work was published in 2009). (In Arabic).

Turki Al-Masaeed, (2017 AD): The challenges of preparing and qualifying teachers in light of the skills of the twenty-first century. The fourth conference, College of Education and Basic Sciences, Ajman - United Arab Emirates. (In Arabic).

Zaitoun, Hassan Hussein. (2005). A new vision in e-learning: concept, issues, implementation, and evaluation. (I 1). Riyadh: The Sound House for Education. (In Arabic). 
